\title{
The Research of the Ability of Fashion Creative Design Talent and Quality Evaluation Model
}

\author{
Jinhua Ruan ${ }^{1}$, Jingjing Zheng ${ }^{1} \&$ Shouzhong $\mathrm{Hu}^{1}$ \\ ${ }^{1}$ Fashion Design and Engineering, Shanghai University of Engineering Science, Shanghai, China \\ Correspondence: Jinhua Ruan, Fashion Design and Engineering, Shanghai University of Engineering Science, \\ Shanghai, China
}

Received: July 10, 2015

Accepted: September 12, 2015

Online Published: November 24, 2015

doi:10.5430/jbar.v5n1p1

URL: http://dx.doi.org/10.5430/jbar.v5n1p1

\begin{abstract}
This paper focus on the ability that fashion creative talent should have. Through literature searching, extracting factors with the pre-survey, researching method, using Spass software to factor analysis and Quadrant law to extract important factor, summary the best training program. Hope to provide a basis for the education of future fashion creative talents, thus promote the improvement of domestic clothing creative talent training mechanism, improve personnel training efficiency and save culture cost.
\end{abstract}

Keywords: Fashion, Creative talent, Costume design, Evaluation model

\section{Introduction}

Currently, there are a lot of research on the cultivating of creative talents, but most of them are just on the education system. There is nearly no study on the nature of clothing creative talents.

For the reform fashion creative talent training mechanism, the most important thing is to understand what kind of people we need, what kind of ability should clothing creative talent have and what kind of ability should we focus on training. Only in this way, can we make the reform on training mechanism sense. Therefore, the most important part of the reform of training mechanism is not mechanism itself, but focus on understanding the point we should educated. The research on clothing creative talent is the basic of institutional reform.

Many abroad training model has been proven very successfully. Reference is not equal to imitate. Blindly imitating only go into misguided, taking more detours. Thus, the most basic of exploring training mode patterns is the local conditions. Therefore, the ability of clothing creative talent should be researched imminently.

Through the summary of the articles about the reform of education system of clothing creative talents, extract what kind of ability should the designer of creative clothing have. Supplement for factors is completed through the pre-study, which is to improve the accuracy of the factors extracted. And then use principal component analysis to analyses extracted factors through research methods, extracting important factor, weights were analyzed, and then comprehensive evaluation is completed by quadrant law. The mode of the ability of clothing creative talent is established.

\section{The Ability of Fashion Creative Design Talent}

The ability of clothing creative talent is extracted by mainly two methods: literature summary; the pre-research.

Creative industry is universally characterized by clothing creative talent, but the clothing creative industries is the particularity of the creative industries. Therefore, clothing creative talent should have the ability, when it is necessary to consider its universality but also consider its particularity. Through the analysis of literature and creative industry, summary characteristics of creative industries, coupled with the analysis of the characteristics of the clothing itself, sum up what ability and quality of clothing creative talent should have: 
A:The basic creative thinking ability and quality

$\mathrm{B}$ :Aesthetic innovation, the challenges of traditional aesthetics

\section{C:Literacy capability}

D:The ability to understanding intellectual property

E:The ability to make sketch into apparel

F:The ability to express language of costume design
Mainly includes four aspects: Innovative color; innovative garment structure; Fabric innovations:

In basis understanding of aesthetics, aesthetic innovation of structure, color, fabric, the courage to express their ideas, the courage to realize their innovation, people's ability, to accept new things, become stronger, given the designer enough space to express themselves

Successful designer must have two aspects of literacy: local culture; international pop culture

Clothing creative industries comprising appearance patents and invention patents, in order to protect their intellectual property and the risk of plagiarism, intellectual property knowledge is necessary

For clothing creative talent, a certain practical ability must be needed; designer' clothing shall comply with the structure of the law; there is the possibility of realization

Expression designed mainly comprising video expression and language expression. the video transmission of information is necessary, so for video clips, or a combination are required mastered. the overall assurance is very important to, including the music choice.

G:The ability of studying consumers' Apparel market determines the designers not to only Psychology have a certain consumer psychology research and grasp international information. The grasp of the international fashion trends is very important.

H:Management skills of apparel product marketing(ability for product operations)

Clothing creative talent should grasp the sales process of apparel to in favor of the designer determining their design direction, to in favor of the promotion of creative clothing, to develop their own creative way garment commodity products business, such as forms of advertising design; advertising.

\section{The Research of the Ability of Fashion Creative Design Talent and the Analysis of the Data}

\subsection{Survey Questionnaire Collective}

The survey questionnaire is conducted according to the ability and quality that costume creative design talent should have (see Annex 1), and then verified questionnaire survey after survey questionnaire form. This study contains three ways: online survey, mail survey and field research in order to obtain sufficient data, so that a more accurate research would be obtained. Research object is garment faculty, students, fashion designers, clothing buyers, clothing design enthusiasts and clothing corporate recruiters to ensure the validity of the questionnaire.

Survey questionnaire use scoring 1-5 points, followed by the importance of the enhanced survey questionnaire eventually recovered 100 copies. 


\subsection{Using Spass Software to Factor Analysis of Research Data}

(1) Reliability and validity analysis

Reliability, use commonly used Cronbach's $\alpha$ reliability coefficient method. validity of law adopted KMO and Bartlett's test. The test results (Table 1) show, Cronbach's $\alpha=0.818>0.8$, indicating that this questionnaire' reliability is very high; KMO test coefficient $>0.5$, ( $\mathrm{x} 2$ statistical significance values Bartlett test of sphericity of probability) $\mathrm{P}$ $=0.000<0.05$, so this questionnaire has construct validity; principal component analysis can be performed.

Table 1 . Reliability and validity

\begin{tabular}{|c|c|c|c|c|c|}
\hline Cronbach's & & & & Sampling & \\
\hline Alpha & & df & 28 & sufficient degree & \\
\hline .818 & $\begin{array}{c}\text { Bartlett test of } \\
\text { sphericity }\end{array}$ & Sig. & .000 & $\begin{array}{c}\text { of } \\
\text { Kaiser-Meyer-O } \\
\text { lkin measure }\end{array}$ & .751 \\
\hline
\end{tabular}

\section{(2) Reduce the dimension}

Eigenvalues and variance contribution table(Table 2);"Total" section of each factor corresponding characteristic root, "variance\% " part of the variance contribution of each factor, the "total\%" part rate. The former can be seen from the table 3 explaining the total variance of 71. 831, and the characteristic values are greater than 1; It is possible to select the first three principal component analysis.

Table 2. Eigenvalues and variance contribution

Initial eigenvalues $\quad$ Extracting square and load

\begin{tabular}{lcccccc} 
Factors & Total & \multicolumn{2}{c}{ Accumulation } \\
1 & 3.603 & 45.032 & 45.032 & 3.603 & 45.032 & 45.032 \\
2 & 1.093 & 13.658 & 58.690 & 1.093 & 13.658 & 58.690 \\
3 & 1.051 & 13.141 & 71.831 & 1.051 & 13.141 & 71.831 \\
4 & .631 & 7.890 & 79.721 & & & \\
5 & .561 & 7.017 & 86.738 & & \\
6 & .500 & 6.252 & 92.990 & & \\
7 & .368 & 4.602 & 97.592 & & \\
8 & .193 & 2.408 & 100.000 & & \\
Extraction Method: Principal component analysis. \\
\hline \multicolumn{7}{l}{} \\
\hline
\end{tabular}

(3) factor extraction

By Table 3 (principal component extraction and analysis of variance decomposition), extract three principal components. Factor A, C, D, E, F, G at a higher load factor 1, a first main component basically reflects information of factors A, C, D, E, F, G; similarly, factor 2 basically reflects the factors information of $\mathrm{H}$; factor 3 reflects the basic essential information of factor B. 
Table 3. Matrix composition

\begin{tabular}{lll}
\hline \multicolumn{4}{l}{ Ingredient } & \\
& 1 & 2 \\
$\mathrm{~A}$ & .719 & .718 \\
$\mathrm{~B}$ & & \\
$\mathrm{C}$ & .741 & \\
$\mathrm{D}$ & .477 & \\
$\mathrm{E}$ & .848 & \\
$\mathrm{~F}$ & .790 \\
$\mathrm{G}$ & .695 \\
$\mathrm{H}$ & \\
Extraction Method: Principal component analysis. \\
a. Three ingredients has been withdrawn.
\end{tabular}

(4) The weight of each factor

Divide the data from the component matrix of Table 3 by the characteristic root corresponding to the principal component, take the square root of the result, the we can get the coefficient corresponding to each indicator of the three principal components. The three principal components are normalized as follows.

$$
\mathrm{F} 1=0.074 \mathrm{~A}+0.142 \mathrm{C}+0.118 \mathrm{D}+0.222 \mathrm{E}+0.220 \mathrm{~F}+0.224 \mathrm{G}
$$

Factor 2 and Factor 3 are expressed by Factor $\mathrm{H}$ and Factor B.

A The basic ability of creative thinking C Cultural literacy D The knowledge of intellectual property E The ability of making clothes according to the fashion design drawing $\mathrm{F}$ The ability of expressing the fashion design drawing by using language $\mathrm{G}$ The ability of researching customer psychology $\mathrm{H}$ The ability of marketing and managing clothing products (The ability of product operation) B Bringing forth new ideas in the field of aesthetics.

\section{The Comprehensive Evaluation and Recommendation of the Results of Statistical Analysis}

Koeffizient corresponding jeder indikator of F1, the first main component, is multiplied by the corresponding contribution rate of component $\mathrm{F} 1$, then divided by the contribution rate of the three extracted three main ingredients, then add Koeffizient corresponding jeder indikator of F2, the first main component, is multiplied by the corresponding contribution rate of component F2, then divided by the contribution rate of the three extracted three main ingredients, then add Koeffizient corresponding jeder indikator of F3, the first main component, is multiplied by the corresponding contribution rate of component F3, then divided by the contribution rate of the three extracted three main ingredients. Normalize the result, and then we can get the integrierte model:

$\mathrm{Y}=0.06 \mathrm{~A}+0.10 \mathrm{~B}+0.11 \mathrm{C}+0.04 \mathrm{D}+0.149 \mathrm{E}+0.162 \mathrm{~F}+0.181 \mathrm{G}+0.207 \mathrm{H}$

In the integrierte model, the coefficient corresponding to each indicator is the weight of each indicator.

(1) The weight of each indicator is as follows (table 4):

Table 4.

\begin{tabular}{llllllll}
\hline $\mathrm{H}$ & $\mathrm{G}$ & $\mathrm{F}$ & $\mathrm{E}$ & $\mathrm{C}$ & $\mathrm{B}$ & $\mathrm{A}$ & $\mathrm{D}$ \\
0.87 & 0.181 & 0.162 & 0.149 & 0.11 & 0.10 & 0.06 & 0.04 \\
\hline
\end{tabular}

(2) The mean value of the weight is $0.125, \mathrm{H}, \mathrm{G}, \mathrm{F}, \mathrm{E}$ are greater than the average, while C, B, A, D are less than the average. Through four-quadrant analysis the factors which have been extracted can be comprehensively estimated. The first quadrant means that a heavier weight has a greater influence on the improvement of clothing designers' 
ability. The second quadrant means that a heavier weight has a less influence on the improvement of clothing designers' ability. The third quadrant means that a lower weight has a less influence on the improvement of clothing designers' ability. The forth quadrant means that a lower weight has a greater influence on the improvement of clothing designers' ability. (Figure 1)

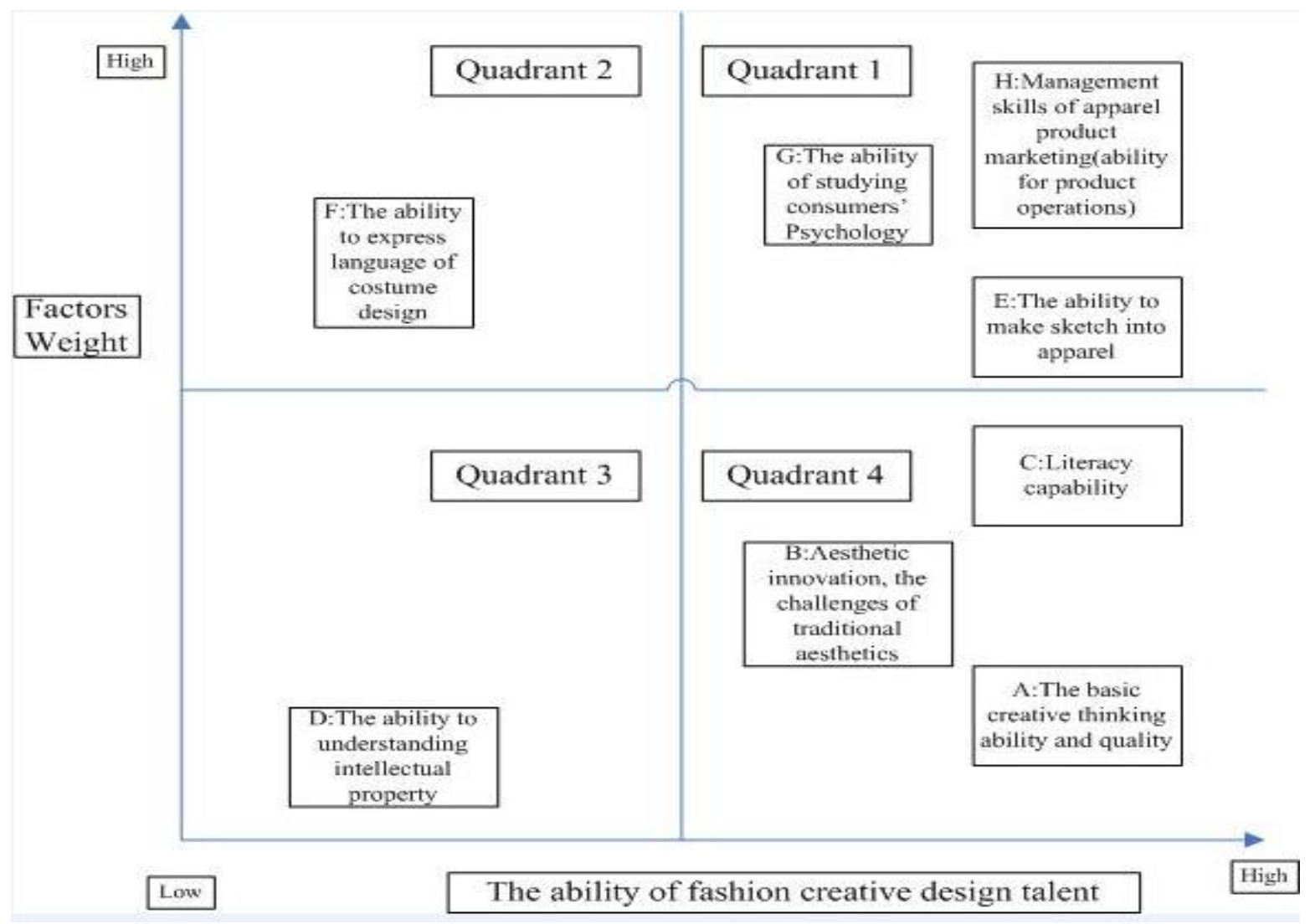

Figure 1. Comprehensive Assessment of Quadrant Model law

Through the quadrantal diagram we can know the influence which each factor about the demands on the ability and qualities of clothing designers has on the improvement of clothing designers' ability. And we can reforms the education system in a well-directed way.

The first quadrant means that a heavier weight has a greater influence on the improvement of clothing designers' ability. H, G, E are located in the first quadrant, which means that $\mathrm{H}$ The ability of marketing and managing clothing products (The ability of product operation), G The ability of researching customer psychology, E The ability of making clothes according to the fashion design drawing are in proportion to the ability and qualities of clothing designers. The improvements of the teaching of those three aspects should be emphasized.

For example, we can cultivate the students' ability of product planning by assigning projects to students and letting them finish by themselves; And we can strengthen the combination of production and academy by letting the students finish enterprise projects individually or by team work; And we can extend the class hours of fashion design courses and practical courses to cultivate students' practical ability, deepen students' knowledge of the structure of clothing and help students have a good command of clothing design.

In the second quadrant, the weight of Factor $\mathrm{F}$ The ability of expressing the fashion design drawing by using language is heavy, and is larger than the average. However, it does not have a great influence on the ability and qualities of clothing designers. So this part can be shortened to save the cultivation cost and time cost in the process of cultivating students.

In the third quadrant, the weight of D The knowledge of intellectual property is low, and its influence on the ability and qualities is little. Students can develop the ability and qualities by themselves.

In the fourth quadrant, the weight of C Cultural literacy B Bringing forth new ideas in the field of aesthetics A The 
basic ability of creative thinking is low. However, they have a great influence on the ability and qualities of clothing designers. They can even promote the development of a certain field.

For example, the mental disorder makes Yayoi Kusama thoroughly accomplished in her design rather than hinder her, and Issey Miyake's innovation on fabrics is second to none. The development of world culture demands the encouragement from more voices. There are many outstanding designers in China, such as Ma Ke-the designer of 'Wu Yong' and 'EXCEPTION', whose innovation on fabrics is widely accepted. She buries the fabrics into the soil. A few days later, she dug out the fabrics and she got the unique fabrics with unique colors. Her behavior isn't random. The willingness to be close to the nature in today's world where the environment is being polluted encourages the success of her design.

So, this part can be said to be the basic of the cultivation of the ability and qualities of clothing designers. The investment in the fourth quadrant should be of the same importance as the investment in the first quadrant. In the process of reforming the teaching, this part should be enhanced.

\section{Conclusion}

For study of what competency should clothing creative talent have, he most important factor were extracted, playing a leading role in the future direction of the culture of clothing creative talent, making the training objectives clear, saving targeted cost culture. However, this article only studied the important factors in theoretically level, hoping cultivating cost and factors could be linked to play a guiding role in training programs in later studies.

For how to cultivate clothing creative talents, the paper $\mathrm{b}$ is only comparing domestic and international creative talent training mechanism, putting forward its own subjective ideas, what the teaching methods should improved. Whether it adapt to domestic education system should have yet to be verified.

\section{Acknowledgements}

This project is sponsored by Shanghai University of Engineering Science 2015-year Innovation Fund for Graduate Students.

\section{References}

Changzhou. (2013). Creative Industry Association. Changzhou Cultural and Creative Industry Development Report.

Jin Yuanpu. (2006). The concept and characteristics of the contemporary world and creative industries, Film Arts, (3), 1-16.

Li Hong Qin. (2005). Emotions Reflect of Clothing Creative Design, Wuhan Institute of Science and Technology. (18), 12, 1-2.

Li Mu. (2014). Comparison China and American Elementary Arts Education, International Educational Exchange, Tsinghua University Research Center of Basic Education, 2014-07-54.

Liao Can. (2008). Creativity China, China Economic Publishing House.

Tang Jie, Yang Jianyun. Teaching Probe on clothing creative design.

Theodore Lewis. (2009). Creativity in technology education: providing children with glimpses of their inventive potential. Int $J$ Technol Des Educ. Department of Work and Human Resource Education. http://dx.doi.org/10.1007/s10798-008-9051-y

Wang Qi. (2009). Nature and Characteristics of "Creative Industries", Economic Theory and Practice, The Cultural Creative Industries. Urban Economy, 2009-03-06.

Ye Li, Hou Xiaoyu. (2012). Funny Innovative Training Model under The Background Creative Iindustries, Discipline Education, Art and Design College, China University of Mining, 2012-07.

Yu Yiqing, Ling Ni. (2011). Thinking and Explore of Chinese High-level Training Model of Creative Design under The International Perspective. Modern Decorative Theory, Zhejiang University of Technology.

Zhan Wangning. (2012). The Transformation Concept of Art Design Talents Education Under the Creative Industrie Economic, China Education Innovation Herald, Design Arts Department of Zheng Cheng College, 2012-No. 14

Zou Hui, Tao Hui. (2010). Teaching Thinking on Costume Design Creative Courses. Shandong Textile Economy, 2010. 11. 